P313 SEXUAL MIXING PATTERNS BY BISEXUALITY AMONG MALE-FEMALE PARTNERSHIPS IN MELBOURNE, AUSTRALIA

${ }^{1,2} \mathrm{~K}$ Greaves*, ${ }^{1,2} \mathrm{C}$ Fairley, ${ }^{1,2} \mathrm{~J}$ Engel, ${ }^{1,2} \mathrm{~J}$ Ong, ${ }^{1}$ E Rodriguez, ${ }^{1,2} \mathrm{~T}$ Phillips, ${ }^{1,2,3} \mathrm{E}$ Chow. ${ }^{1}$ Melbourne Sexual Health Centre, Alfred Health, Melbourne, Australia; ${ }^{2}$ Central Clinical School, Faculty of Medicine, Nursing and Health Sciences, Monash University, Melbourne, Australia; ${ }^{3}$ Centre for Epidemiology and Biostatistics, Melbourne School of Population and Global Health, The University of Melbourne, Melbourne, Australia

10.1136/sextrans-2021-sti.368

Background Past studies have shown that sexually transmitted infections (STI) may be transmitted across different high-incidence populations in Australia. Bisexual individuals have a higher likelihood of STI transmission between different populations due to their sexual practices. This study aimed to explore the sexual mixing patterns by bisexuality among malefemale partnerships attending a sexual health clinic in Melbourne, Australia.

Method We analysed all male-female partnerships attending the Melbourne Sexual Health Centre (MSHC) together over a 5 -year period from 2015 to 2019. Gender and number of sexual partners were collected via computer-assisted self-interview (CASI). We calculated the proportion of partnerships where at least one individual reported bisexual activity (i.e., having both same-sex and opposite-sex partners in the previous 12 months).

Results A total of 2112 male-female partnerships (i.e. 4224 individuals) were included with a median age of 27 years (IQR 23-31) and 37.5\% (1584/4224) were born in Australia. Overall, $89.3 \%(1885 / 2112)$ of male-female partnerships were heterosexual partnerships where both individuals only had opposite-sex partners; however, there were 9.5\% (201/ 2112) of partnerships where one of the individuals was bisexual, and $1.2 \%(26 / 2112)$ of partnerships where both individuals were bisexual. These proportions did not change between 2015-2019. Bisexual men had a higher number of casual sex partners than heterosexual men (median $=5$ vs 1 ; $\mathrm{p}<0.001$ ). Similarly, bisexual women had a higher number of casual sex partners than heterosexual women (median $=6$ vs $1 ; \mathrm{p}<0.001)$.

Conclusion One in 10 male-female partnerships had at least one individual who reported bisexual sexual activity within the previous 12 months, and bisexual individuals had more casual sex partners than heterosexual individuals. The sexual mixing by bisexuality in male-female partnerships may have significant impact on STI transmission in the heterosexual populations.

\section{P314 RISK OF INFERTILITY IN RELATION TO CHLAMYDIA AND PELVIC INFLAMMATORY DISEASE: A CASE-CONTROL STUDY IN CHINESE POPULATION}

L Liu*, W Tang, C Li, X Sun, J Liu, C Wang, B Yang. Dermatology Hospital of Southern Medical University; Institute for Global Health and Sexually Transmitted Infections, Southern Medical University, Guangzhou, China

10.1136/sextrans-2021-sti.369

Background Chlamydia trachomatis and pelvic inflammatory disease (PID) are well-known risk factors for female infertility. But there are limited evidence from China. This study aimed to estimate the association between previous/current chlamydia infection, PID and infertility in Chinese population.
Methods We performed a 1:2 matched case-control study with two control groups: pregnant controls and non-pregnant controls in China in 2019. Women diagnosed with infertility were selected as cases $(n=255)$. Controls were selected based on the following criteria: Pregnant women who were documented in the selected hospitals of the study cities were selected as Pregnant controls $(n=510)$, and people who sought for health care in Obstetric/Gynecologic clinics, Family Planning clinics, Dermatology \& STD Department or Urological department for the first time in the past one year were selected as Non-pregnant control $(n=510)$, reported ever having sexual intercourse and willing to be tested for chlamydia. Infertility induced by male factors and people who used antibiotic in vagina within two weeks were excluded. First-stream specimen of urine samples were tested for chlamydia by nucleic acid amplification testing (NAAT). Conditional logistic regression was used to estimate the association.

Results The prevalence of previous chlamydia infection and PID were significantly higher in cases $(2.4 \%, 17.3 \%)$ than in controls (Non-pregnancy: 0.4\%, 3.0\%; Pregnancy: $0.4 \%$, 9.0\%). The current chlamydia infection rates were $5.9 \%$, $7.3 \%$ and $7.1 \%$ in infertile, pregnant and non-pregnant women, respectively. After adjusting for maternal age, BMI, monthly income, chronic disease and other genital tract infection, PID largely elevated the risk of infertility (Non-pregnancy: adjusted $\mathrm{OR}=2.41,95 \% \mathrm{CI}: 1.47,3.95$; Pregnancy: adjusted $\mathrm{OR}=6.67,95 \% \mathrm{CI}: 3.42,12.98)$, while past chlamydia infection was marginally associated with infertility.

Conclusion Previous chlamydia infection was marginally associated with infertility, while previous PID was indicated to largely elevate the risk of infertility.

\section{P315 INCREASED CLINICIAN CONFIDENCE AND UPTAKE OF EVENT-BASED PREP BY MEN WHO HAVE SEX WITH MEN DURING THE COVID-19 PANDEMIC}

D Williams*, E Buck, A Parkhouse, D Richardson. Brighton and Sussex University Hospitals NHS Trust, Brighton, UK

10.1136/sextrans-2021-sti.370

Background HIV pre-exposure prophylaxis (PrEP) is an effective, safe strategy to prevent HIV. PrEP can be used either daily or as an event based dosing (EBD) regimen by men who have sex with men (MSM) having condom-less anal sex, however clinicians with expertise delivering daily PrEP often lack confidence delivering EBD-PrEP. During the COVID-19 pandemic, MSM appear to have tailored their sexual behaviour in-line with local social restrictions including the way they use PrEP.

Method We aimed to investigate the proportion of MSM using EBD-PrEP between October-December 2020 and to survey clinician confidence in delivering EBD-PrEP through an online questionnaire.

Results 551 MSM were seen who were eligible for PrEP in the study period, of which 448 were prescribed PrEP (64declined, 2-stopped, 8-new patients and 29-repeat attenders accessed PrEP from another source). The median age of PrEP users was 37 years (IQR=29-48). Overall, 94/448 (21\%,95\% $\mathrm{CI}=17-25)$ of MSM were using EBD-PrEP. New starters were significantly more likely to use EBD-PrEP compared to existing PrEP users (34\%.v.13\%, $\square 2=27.6, \mathrm{p}<0.00001)$. There was no significant difference in age between daily and EBD-PrEP 